\title{
Una visión global de las lenguas criollas: perspectivas y retos de la criollística ${ }^{1}$
}

\author{
A global vision of creole languages: perspectives \\ and challenges of Creole studies
}

Javier Enrique García León ${ }^{2}$

\section{Resumen}

El objetivo del presente artículo es analizar los diferentes postulados que se han desarrollado acerca de las lenguas criollas con el fin de obtener una visión global de este fenómeno. En la primera parte, se estudian las diferentes definiciones existentes de los pidgins y los criollos al igual que sus clasificaciones. Posteriormente, se analizan las principales teorías acerca del surgimiento de estas lenguas para luego mirar sus características principales. En la tercera parte, se cuestionan aspectos como el continuo lingüístico poscriollo y la descriollización a la luz de nuevos estudios. Finalmente, se hace un breve análisis de la criollística como disciplina y se plantean los nuevos retos que tendrá que afrontar. Se concluye que las lenguas criollas son sistemas de comunicación particulares debido a su forma de surgimiento y características lingüísticas, además se determinó que es necesario reevaluar algunos elementos teóricos que han generado un entendimiento parcial del fenómeno lingüístico aquí descrito. Se espera que este artículo contribuya a la disciplina de lenguas en contacto, específicamente al estudio de las lenguas criollas, y genere debates teóricos que ayuden a un mejor entendimiento de este tipo de códigos lingüísticos.

Palabras claves

Criollística, lenguas criollas, continuo lingüístico poscriollo, descriollización, varialingüísmo, lenguas en contacto.

\section{Abstract}

The objective of this article is to analyse the different hypotheses which have developed around creole languages with the intention of gaining a global vision of this phenomenon. In the first part, the varying definitions of pidgin and creole languages currently in existence will be examined. Following this, the principal theories around the development of these languages will be analysed in order to look at their principal characteristics. In the third part, aspects such as post-creole linguistic continuum and de-Creolisation will be examined, taking into account new studies. Finally, a brief analysis of Creole studies as a discipline is conducted and we consider new challenges which must be confronted. It is concluded that creole languages are particular communication systems due to the way in which they developed and also to their linguistic characteristics. Furthermore, it is determined that it is necessary to re-evaluate certain theoretical elements which have given rise to a partial understanding of the linguistic phenomenon described here. It is hoped that this article will contribute to the field of language contact, and specifically to the study of creole languages, and that it will generate theoretical debates which will help to achieve a better understanding of this type of linguistic codes.

\section{Key words}

Creole studies, creole languages, post-creole linguistic continuum, de-Creolisation, varialanguage, language contact.

Artículo recibido el 24 de enero de 2012 y aprobado el 7 de febrero de 2014

1 Agradezco a la profesora Olga Ardila del Departamento de Lingüística de la Universidad Nacional de Colombia por sus valiosos aportes durante el curso de Teoría 1 de la Maestría en Lingüística.

2 Universidad Nacional de Colombia, Bogotá, Colombia. jaegarciale@unal.edu.co 
Universidad Pedagógica Nacional

Facultad de Humanidades

El cambio lingüístico ha sido uno de las áreas más estudiadas por la sociolingüística. Se han analizado elementos sociales, históricos e incluso diafásicos que generan cambios en las lenguas. Uno de los factores sociales es el contacto de lenguas. Cuando dos lenguas entran en contacto se generan cambios profundos en las estructuras lingüísticas que pueden ser enriquecedores para las lenguas que entran en relación (Weinreich y Martinet, 1963; Siguan, 2001). Sin embargo, dicho contacto no solo genera modificaciones lingüísticas, también es posible que, llevado al extremo, genere nuevas lenguas. Estas nuevas creaciones lingüísticas han sido denominadas pidgins o criollos dependiendo de características y elementos sociohistóricos y sociolingüísticos particulares. Dentro de la subdisciplina denominada lenguas en contacto, entonces, encontramos a aquellos investigadores que se ocupan de estas lenguas dando origen a una subdisciplina de la lingüística, la criollística (Herzfeld, 2002). De acuerdo con Patiño (2002), es a partir de los años sesenta cuando se empezó a consolidar esta subdiciplina, haciendo que las lenguas criollas fueran de particular interés para lingüistas, antropólogos e historiadores. De este modo, el presente artículo tiene como objetivo principal presentar los planteamientos centrales que esta disciplina ha elaborado durante los últimos años sobre las lenguas criollas.

Las lenguas criollas, como tipos de lenguas, fueron consideradas degeneraciones o desviaciones de otros sistemas lingüísticos (Hymes, 1971; Bettina Migge, Léglise, y Bartens, 2010). Estas concepciones, ya rebatidas por la criollística, aún circulan en algunos países donde existen estas lenguas. Es común que los hablantes usen términos como broken english, bad english, y dialect (en forma peyorativa) para referirse al criollo de base inglesa. Es de suma importancia entender qué son estas lenguas realmente, cómo se originan, y qué procesos históricos y sociales conllevan, pues así podrán ser puestos en juicio los estereotipos lingüísticos que se tienen sobre ellas. Aunque muchos criollistas, entre ellos Hymes (1971), Bickerton (1973), Siegel (2006), Alleyne (1994) y Patiño (2002), ya han desmitificado algunas de estas actitudes negativas, también es cierto que en aras de entender este fenómeno se han generado imprecisiones y generalizaciones que, en algunas ocasiones, conllevan a un entendimiento parcial o sesgado de este fenómeno (Jourdan, 1991). Por esto, es de suma importancia volver a mirar los planteamientos centrales que se han hecho sobre las lenguas criollas, sobre todo los más recientes, pues de este modo se obtendrá una visión global del fenómeno sin desconocer las particularidades de cada lengua y situación social. Además, poner en dialogo diversas perspectivas teóricas con investigaciones empíricas genera nuevos interrogantes y horizontes para esta disciplina. Para esto, este texto se estructura de la siguiente manera: primero, se definen las lenguas criollas y los pidgins, y se plantea una clasificación a la luz de los autores que se han ocupado del tema. En segundo lugar, se analizan las principales teorías acerca del surgimiento de estas lenguas. En la tercera parte se miran las características principales de los criollos y se cuestionan conceptos como el continuo lingüístico poscriollo y la descriollización, y en la cuarta y última sección, se hace un breve análisis de la criollística como disciplina y se plantean algunos retos para futuras investigaciones y trabajos teóricos en este campo.

\section{Pidgins y lenguas criollas}

\section{Definiciones}

Según autores como Decamp (1971), los primeros estudios definen a las lenguas criollas como dialectos mezclados de una lengua estándar, usualmente europea, como el inglés, el francés, el español y el portugués. Estas son definiciones pobres, pues se limitan a las similitudes de vocabulario que existen entre los criollos o pidgins con una lengua europea. Pero aún más grave, el término de mezcla ha llevado a que estas lenguas sean consideradas como un popurrí, es decir, como lenguas sin una estructura uniforme y poco genuina. Teniendo en cuenta que los pidgins y los criollos están íntimamente relacionados, se puede afirmar, desde una mirada más amplia, que surge un pidgin cuando entran en contacto dos comunidades que no comparten un 
idioma común y por ello dichas comunidades necesitan una forma de comunicación (Patiño, 2000). Es decir, el pidgin es un código de emergencia que se origina cuando hay una necesidad imperiosa de comunicarse entre dos comunidades que no comparten un código lingüístico. Por lo general, dichos pidgin surgen en situaciones de contacto producidas por desplazamientos masivos de humanos, la llegada de africanos al nuevo mundo, por ejemplo, o en situaciones de frontera o inmigración. El contacto se puede dar entre grupos cuya lengua y comunidad tienen prestigios diferentes, alto y bajo, o entre grupos con el mismo prestigio (Romaine, 1988).

Un rasgo importante de los pidgins es que al ser un instrumento auxiliar de comunicación no son lenguas maternas de ninguno de los grupos que entran en contacto, hecho que lo diferencia, por lo menos a nivel teórico, de los criollos. Dada esta circunstancia de origen, los pidgins se caracterizan por un número limitado de vocabulario y por la eliminación de diversos mecanismos gramaticales como el género y el número (Decamp, 1971), aspecto que se ha denominado simplificación. Estos códigos de contacto pueden ser pasajeros o, como afirma Patiño (2000a), pueden afianzarse socialmente y ampliar progresivamente sus recursos lingüísticos. El elemento esencial en la relación pidgin y lengua criolla es la nativización. Este es el paso crucial que conduce del pidgin a una lengua criolla y sucede cuando la lengua de contacto se convierte en la lengua materna de los grupos interactuantes. Para Patiño (2000a), es necesario que la lengua criolla cumpla funciones comunicativas mucho más amplias y tenga una estructura gramatical mucho más completa al igual que un léxico mucho más rico que el del pidgin. Para que esto suceda se deben dar una serie de factores sociales:

En los casos en los que el contacto incluye un idioma de prestigio que actúa como modelo para que surja el criollo, debe haber un contexto de marcado distanciamientos social entre la clase dominante y el grupo o grupos subordinados, solo así se dan las condiciones, de imposibilidad de correcto aprendizaje de la lengua modelo por parte de la población socialmente inferior y la solución es el criollo. (Patiño, 2000, p. 125)
Un ejemplo típico de este proceso es el que se llevó a cabo en las plantaciones (Patiño, 2000b) y (Mintz, 1971). En este contexto existían lenguasmodelo de los patronos colonos que no podían ser fácilmente aprendidas debido a la separación social que existía entre los esclavos o trabajadores y los colonizadores; además tampoco era posible aprender ninguna de las lenguas de la población subordinada por estar todas en situación de inferioridad, entonces, el grupo en contacto recurrió a la formación de un vernáculo criollo.

El concepto de nativización ha sido un elemento central en la criollística como ya se mencionó. No obstante, son varios los autores (Romaine, 1988; Salikoko, 2006; Jourdan, 1991) que consideran que esta etapa no es el elemento central para pasar de un pidgin a un criollo. Los aportes de Jourdan (1991) esclarecen algunos planteamientos que han llevado a un entendimiento parcial de este fenómeno. Para este autor, la propuesta arriba expuesta se limita a ver el criollo como el resultado exitoso de la transformación de un pidgin en una lengua natural, por consiguiente la transformación de un pidgin en un criollo es vista como un proceso evolutivo; sin embargo, no es claro si ambas lenguas pueden coexistir en la misma sociedad o si la lengua criolla puede existir antes de convertirse en la lengua materna de un grupo de niños. El caso del pidgin de Melanesia en Oceanía es bastante importante para clarificar esta problemática, aunque autores como Patiño (2002) lo tratan como una excepción, este pidgin nos enseña dos cosas frente a la nativización. Primero que en esta región se encuentran pidgins mucho más elaborados y estables de lo que se supone son estas lenguas, además, estas lenguas se han convertido en las lenguas maternas de una generación de niños (Jourdan, 1991). Asimismo, los pidgins lexicalizados por el inglés han servido como lenguas francas en plantaciones, mercados y relaciones interétnicas en los últimos 120 años, y solo recientemente han comenzado un proceso de criollización, esto quiere decir que por lo menos hay 5 generaciones de hablantes de pidgin que tienen una lengua materna diferente. Esto significa que el pidgin no necesariamente debe pasar por un proceso de nativización o desaparecer, este puede seguir cumpliendo sus funciones durante varias generaciones. 
Universidad Pedagógica Nacional

Facultad de Humanidades

Otra diferencia central es que el pidgin de Melanesia ha sufrido una expansión sintáctica radical, contradiciendo la idea tradicional de que los pidgins son "very simple grammatically, unregularized, and unstable" (Bickerton, 1976, p. 197). A la par, donde se ha dado la criollización (desde la perspectiva tradicional de nativización) poca expansión gramatical se ha observado en comparación con la del pidgin (Jourdan, 1991). De este modo, parece un poco difícil usar teorías lineales acerca del proceso de criollización, es decir, como la simple transformación de pidgins a criollos. Entre otras razones porque así como es posible que la criollización se dé en cualquier lugar en el ciclo de vida pidgin/criollo, también es posible que los criollos existan sin pasar obligatoriamente por la etapa de pidginización, además, los pidgin pueden extenderse sin nativizarse por bastante tiempo. Al respecto, y basado en la situación sociolingüística de Belize, Jourdan afirma: "There are pidgins and creole varieties of the same language existing simultaneously in the same sociolinguistic niche or in different niches. This simultaneous existence of pidgins and creoles varieties is linked to the sociolinguistic praxis of the speakers" (Jourdan, 1991, p. 194). De este modo, se puede afirmar que la nativización es un elemento concomitante de la criollización, pero no su aspecto definitorio. Para Jourdan lo fundamental en el estudio de la criollización de una lengua es la aparición de contextos sociales que obligan a los hablantes de pidgin a manejar una lengua universal, una lengua que sirva para todos los propósitos de la comunicación. Entonces, para que una generación de niños crezca como hablantes de criollo debe existir un contexto en el que el pidgin sea la lengua de la comunidad de habla; esto es, donde la comunidad dependa del pidgin para la comunicación diaria (Jourdan, 1991; (Bakker, 2008); de esta manera, el pidgin deja de ser una lengua franca que solo sirve para ciertas funciones de la comunicación, como el comercio. Además, si los padres sienten que deben criar a sus hijos en este pidgin, y si los niños usan esta lengua fuera del círculo familiar, es porque en ese instante esa lengua se ha convertido en un elemento vital para la comunidad. Para concluir, lo que permite distinguir entre un pidgin y un criollo es la práctica lingüística de los hablantes: un pidgin es una lengua secundaria en una comunidad de habla y el criollo es una lengua principal, con o sin nativización (Jourdan, 1991). De este modo se deja atrás la oposición tradicional entre segunda lengua y lengua materna.

\section{Clasificación y localización}

Las lenguas criollas han sido generalmente clasificadas a partir de sus lexificadores, es decir, de la procedencia de su vocabulario. De este modo, existen lenguas criollas de base léxica, portuguesa, española e inglesa entre otras (Holm, 2000), (Patiño, 2000a). Es importante notar que esta clasificación ha llevado a algunos mal entendidos, pues como afirma Decamp (1971), se cree que los criollos son variedades dialectales de estas lenguas europeas por compartir la mayor parte del vocabulario con ellas; sin embargo, la lexificación debe ser entendida únicamente como el proceso de sustitución de vocabulario en donde se adopta la entrada léxica de la lengua meta (Muysken, 1981). Así, la relación entre la lengua europea de lexificación y los criollos no es de carácter genealógico, sino de suministro de vocabulario y de algunos elementos gramaticales. Con esta aclaración, se puede afirmar que se hablan criollos de diferente base léxica en América, África, Asia y Oceanía, siendo la región del Caribe el lugar donde se concentra el mayor número de lenguas criollas. En los vernáculos ingleses del atlántico se distinguen cinco subgrupos: a) criollos de África occidental, b) de Surinam; c) de Norte América, d) del Caribe oriental ye) del Caribe occidental. Por su parte en la rama del pacifico, existen variedades como el pidgin english de las costas de China, los criollos de Hawái y Australia, y el pidgin english de Melanesia (John Holm, 1989; Patiño, 2000). No obstante, el tamaño de muchas comunidades de habla criolla milita en contra del reconocimiento de los pidgins o criollos, pues en una comunidad lingüística pequeña son más fácilmente considerados como representantes de una variedad dialectal de un grupo más grande, este es el caso del Wutun en la provincia Qinghai de China (Muysken y Veenstra, 1995). 
En la zona más prolífera en cuanto a lenguas criollas se refiere, encontramos criollos de base léxica inglesa, francesa, y española. Entre los actuales territorios de habla criolla inglesa encontramos las islas de Trinidad y Tobago, Grenada, Barbados, San Vincente, Santa Lucia, Dominica, Monserrate, San Kitts y Nevis, Antigua y Barbuda, Anguila, Los Turks y Caicos, las diversas islas de las Bahamas, Jamaica e Islas Caimán (Aceto, 2006). Sumados a estos criollos encontramos los vernáculos de las Islas Misquitos en Honduras, la isla de Rama Cay en Nicaragua, Belice o la antigua Honduras británica, las Bocas del Toro, Colon y Panamá City en Panamá y el criollo de San Andrés y Providencia de Colombia (Herzfeld, 2008). Las zonas de habla criolla francesa son Haití, Luisiana, la Guyana Francesa, Martinica, Guadalupe, Marie Galante, Dominica, Santa Lucia, entre otras. Los dos únicos criollos de base léxica española son el palenquero colombiano y el papiamiento de las Antillas Holandesas (Patiño, 2000). De este modo queda en evidencia la riqueza lingüística de la región del Caribe, lugar obligado para el estudio de estas lenguas.

Desde un enfoque sociohistórico, la clasificación de los criollos se da desde dos puntos centrales: los datos demográficos, sobre todo aquellos en relación con el número de afrodescendientes y europeos en un lugar específico; y la estructura socioeconómica de los escenarios donde se dieron los contactos lingüísticos (Chaudenson, 1977). De este segundo punto surgen dos tipos de criollos: los endógenos y los exógenos. Los primeros son aquellos cuya población de hablantes no sufrió desplazamiento sino que permaneció en su hábitat original, como fue el caso de los criollos de base inglesa y portuguesa que se formaron en las costas de África occidental. El eje socioeconómico de dichas poblaciones era el comercio y su composición étnica permaneció homogénea, en términos generales pudieron conservar su cultura. Por su parte, los criollos exógenos son aquellos que se originaron por desplazamientos masivos de poblaciones que tuvieron que dejar sus lugares de origen como es el caso de los afrodescendientes del Caribe, cuyo contexto socioeconómico fueron las plantaciones, una estructura socioeco- nómica nueva que tenía por objetivo aculturizarlos (Chaudenson, 1977).

\section{El origen de las lenguas criollas, perspectivas y debates}

Una de las preocupaciones centrales de la criollística es la de elucidar las cuestiones referentes al origen de las lenguas criollas. Muchos autores han establecido diversas hipótesis que tratan de entender cuál fue la génesis de estas lenguas, dichas hipótesis han sido elaboradas por lo general a partir de la relación entre la lengua criolla y su sustrato o su superestrato. Sin embargo, es difícil determinar cuál perspectiva es la más adecuada; aquí nos centramos en la clasificación hecha por Baker y Mühlhäusler (2006) por cubrir los aspectos centrales del origen de estas lenguas. La primera perspectiva, de superestrato y algunas veces denominada teoría de origen europeo, considera que los criollos esencialmente se originan de variedades regionales no estandarizadas de lenguas europeas, pues los primeros esclavos adquirieron aproximaciones cercanas a las variedades regionales hablada por la población europea presente en las colonias y la transmitieron al próximo grupo de esclavos que llegaron. De este modo, el segundo grupo de esclavos adquirió esta variedad del primer grupo con algunas modificaciones, así, los criollos son asumidos como variedades de una lengua europea (Migge, 2003). Sin embargo, esta teoría no tiene en cuenta los diversos elementos históricos que llevaron a la conformación de las lenguas ni puede explicar la notoria semejanza estructural que se da entre las lenguas criollas de diferente base léxica.

La segunda gran teoría es la denominada de sustrato. Esta tiene por objetivo identificar elementos de lenguas africanas en las lenguas criollas, incluso llegando a plantear la perspectiva monogenética que considera que gran parte de los pidgin y criollos del planeta tuvieron un único origen, un protopidgin afroportugués que se habría utilizado en las factorías esclavistas de África occidental desde el siglo xv y que se habría expandido por el mundo a partir de la trata de esclavos africanos al nuevo mundo. Esta perspectiva tiene bastantes seguidores, pues permite 
Universidad Pedagógica Nacional

Facultad de Humanidades

explicar la similitud estructural que se encuentra entre diversos pidgins y criollos de diferentes latitudes (Patiño, 2000). De este modo, las estructuras gramaticales habrían sido establecidas con el pidgin original, es decir, las estructuras léxicas y gramaticales similares serían retenciones del periodo común de origen previo a la relexificación. Hancock (1971) considera que el proceso llevado por estas lenguas no es la relexificación, o el simple remplazo de vocabulario, sino una construcción de léxico o supralexificación. Este proceso puede ocurrir en tres casos (a) cuando un elemento léxico entra en desuso, (b) cuando se retienen las dos palabras, la vieja y la nueva, con el mismo significado y (c) cuando se especializan ítems léxicos.

Las críticas que se han hecho a la perspectiva anterior se basan principalmente en el innatismo chomskiano, por ello estas críticas se consideran pertenecientes a una perspectiva universalista. Entre sus fundadores se encuentra Bickerton (1984) quien luego de realizar investigaciones en Guyana se trasladó a Hawái para estudiar el criollo de base léxica inglesa de esta región. Allí encontró una serie de elementos similares a los de los criollos caribeños, sin embargo, en esta región, no hubo presencia africana; esto llevó a Bickerton a crear la teoría unificada de la adquisición del lenguaje donde considera que la pidginización y la criollización no son procesos misteriosos ni únicos (Veenstra, 2008), sino que se deben a un proceso de adquisición del lenguaje en unas circunstancias particulares respecto al acceso que los hablantes tienen de la lengua de superestrato. Para Bickerton (1981) el escenario de adquisición fue el siguiente, los esclavos fueron traídos desde diferente lugares hablando una seria de lenguas ininteligibles para trabajar en las plantaciones, esto hizo que las instrucciones fueran dadas en una lengua europea; por consiguiente, estos esclavos adquirieron algo del vocabulario europeo a partir del cual trataron de construir enunciados basados en la gramática de su primera lengua. La pidginización, desde la perspectiva de Bickerton, es un proceso de aprendizaje de una segunda lengua por parte de un grupo en esta situación de distanciamiento social. De este modo, la criollización es también un proceso de aprendizaje, pero esta vez de la lengua materna en condiciones de difícil acceso al modelo lingüístico (Aceto, 1999) que se da reestructurando el pidgin para que cumpla las funciones de un idioma nativo; dicha restructuración se debe en gran parte al bioprograma del lenguaje humano que es el conjunto de reglas y estructuras innatas del lenguaje (Baker y Mühlhäusler, 2006). De este modo, Bickerton explica las similitudes que se dan entre los diversos criollos del Caribe y el de Hawái, sus elementos análogos no se deben, como lo planteaba la perspectiva sustrastista, a una lengua común de origen, sino a las estructuras innatas de los hablantes.

Para autores como Jourdan (1991), nuevos elementos históricos y lingüísticos están cambiando la forma de ver el origen de las lenguas criollas, pues se tiene más información sobre las lenguas africanas relacionadas con los criollos y los contextos sociohistóricos que llevaron a la formación de pidgins. Este autor considera que la teoría del bioprograma debe ser examinada con cuidado en la medida en que no es del todo posible pensar que la mayoría de los hablantes consideraban la lengua de superestrato como el modelo a aprender, existieron hablantes que no estaban interesados en hablar dicha lengua, pero que sin embargo desarrollaron el pidgin. Además, no se puede concebir el pidgin únicamente como el resultado de un aprendizaje pobre de la segunda lengua a causa de la distancia social. Por ejemplo, estudios recientes sobre las circunstancias históricosociales de algunos criollos demuestran que la división por clases no fue netamente entre dos polos opuestos: blancos y negros; sino que existieron subgrupos con un acceso no restringido a la lengua de superestrato (Arends, 1995), es decir, no se dio un aprendizaje limitado. En conclusión, es posible afirmar que las perspectivas basadas en un solo factor no pueden explicar el origen de las lenguas criollas, y por esto es fundamental empezar a buscar soluciones que logren combinar los diversos agentes que intervienen en este fenómeno.

\section{Características generales de las lenguas criollas, la descriollización}




\section{y el varialingüismo}

De acuerdo con Patiño Rosselli, una caracterización adecuada de las lenguas criollas debe señalar principalmente las circunstancias sociohistóricas de su origen, pues son estos elementos los que distinguen estas hablas de otros códigos lingüísticos (Patiño, 2002). En primer lugar, estas lenguas no forman parte de una trayectoria de evolución lingüística, como las lenguas romances, sino que se originan en determinados lugares y momentos. Además, su surgimiento se produce siempre en un marco sociohistórico caracterizado por el encuentro de comunidades con una lengua y cultura diferentes. Este encuentro es lo que Muysken y Veenstra (1995) denominan "el desarrollo lingüístico violento", haciendo referencia a los problemas de comunicación al que se vieron enfrentadas las comunidades en contacto. Por otra parte, aplicar las técnicas de la lingüística histórica en los criollos resulta problemático, pues genera interrogantes que no se pueden responder como por ejemplo icon qué lengua se debe comparar el criollo para entender su origen, con la lengua europea que lo lexificó o con la lengua africana de la que tomo la mayoría de los rasgos gramaticales? (Muysken y Veenstra, 1995). Esto demuestra que los criollos no pueden ser caracterizados a partir de la lingüística histórica, ya que, como se mencionó, dependen de hechos sociales específicos: los encuentros interétnicos e interlingüísticos.

Dado este tipo de surgimiento, se puede afirmar que la característica central de los criollos es su carácter mixto. Estas lenguas cuentan con elementos de las lenguas nativas (substrato), de las lenguas del país, por lo general europeas (superestrato), y algunas veces de las lenguas con que han convivido o conviven (adstrato) (Patiño, 2002). Sin embargo, no se pueden limitar los criollos a la suma de estas lenguas, pues se deben más a un proceso de reajuste e innovación. Dicho proceso de reajuste, ha generado, a nivel lingüístico, tres características centrales que se dan en la mayoría de los criollos, sobre todo en los del atlántico: primero, una sencillez en los medios expresivos, sobre todo a nivel fonoló- gico, pues se prefieren esquemas silábicos sencillos. Segundo, estas lenguas tienden a la separación de los conceptos gramaticales en palabras o partículas independientes, hecho denominado organización analítica. Tercero, la mayoría de las lenguas criollas tienen un vocabulario proveniente de una lengua colonizadora como el español, el inglés, el francés o el portugués (McWhorter, 1998). Cabe mencionar que estas características no deben ser vistas negativamente, como algunas veces se ha hecho, pues al hablar de sencillez nos referimos a una reestructuración lingüística diferente en comparación con las lenguas de tradición histórica. La sencillez de ninguna manera indica que los criollos son inferiores lingüísticamente, pues los hablantes de estas lenguas pueden utilizarlas de manera efectiva para cualquier fin en cualquier contexto comunicativo (Romaine, 1996).

Si miramos los criollos desde una perspectiva sociolingüística y no únicamente lingüística, encontramos que por lo general estas lenguas conviven con otros idiomas, casi siempre los idiomas dominantes de los que tomaron el léxico. Esta convivencia en muchas ocasiones genera situaciones de diglosia, pues hay una lengua principal, de mayor prestigio y en algunos casos oficial, que se usa para las funciones oficiales, y la lengua criolla inferiorizada que se restringe a funciones familiares y cotidianas, no usada en circunstancias formales (Patiño, 2000a; Donald Winford, 1994). En estos casos diglósicos, las fronteras entre ambos códigos están social y psicológicamente marcadas, tanto así que los hablantes bilingües son los que tienen el criollo como lengua materna, pues deben usar ambas lenguas en distintos ámbitos sociales. Dicha convivencia, además, ha llevado a que las lenguas criollas tengan un grado mayor de variación, ya que al estar en contacto con otros idiomas, su fonología y gramática varían más que las de las lenguas dominantes. Cabe mencionar que existen algunos criollos que gozan de prestigio al menos como lenguas nacionales, por ejemplo en Haití y Jamaica (Doucet, 2000); sin embargo, son muy pocos los que gozan de este beneficio. 
Universidad Pedagógica Nacional

Facultad de Humanidades

Este contacto entre la lengua dominante y el criollo ha llevado a hablar del continuo lingüístico poscriollo y la descriollización. El concepto de continuo lingüístico poscriollo se le ha atribuido a Bickerton (1973); sin embargo, este término se debe a los primeros estudios realizados por Bailey (1966) sobre el criollo jamaiquino y luego retomados por Decamp (1971). La primera autora afirma que los hablantes jamaiquinos son capaces de pasar del criollo al inglés estándar y viceversa en un solo enunciado, hecho que hace muy difícil delimitar si se trata de un criollo o de la lengua estándar. Por su parte, el segundo autor considera que si es posible delimitar dicho cambio a partir de una serie de elementos lingüísticos presentes o no en las producciones de los hablantes; la alta o baja presencia de dichos elementos, entre ellos la eliminación de la copula, permitiría determinar si se trata del criollo o de la lengua estándar. Decamp (1971) también afirma que una sociedad poscriolla debe cumplir con dos características para asumir dicha denominación: primero, la lengua dominante en la comunidad debe ser la misma que la lengua que dio la base léxica al criollo, pues si es diferente lo más probable es que el criollo se mantenga estable; $y$ segundo, el sistema social de estas comunidades debe permitir movimiento social para que los hablantes criollos se vean inducidos a hablar el estándar.

Por su parte, Bickerton (1976) hace un estudio del criollo de Guyana a la luz de los postulados de Decamp donde afirma que los hablantes guyaneses han descriollizado su habla de acuerdo con su nivel de educación, lugar de trabajo y localización; dicha descriollización es lo que ha llevado a un continuo. De este modo, la descriollización sería la atracción del habla criolla por parte del idioma de superestrato debido a los elementos mencionados, este es el caso de la atracción del inglés sobre el criollo jamaiquino; en tal situación el resultado es un continuo de variedades que se diferencian entre sí gradualmente (Patiño, 2000a). El continuo sería, entonces, el espectro de estas variedades que va desde (a) el acrolecto o la modalidad más cercana lingüísticamente a la lengua de superestrato, (b) al basilecto, la más alejada del modelo estándar y por consiguiente la que representa el criollo "puro", pasando por un (c) mesolecto o etapa intermedia. Bickereton además asume que lo encontrado en Guyana y posteriormente en Hawái es verdad para todas las hablas criollas en las que la lengua estándar europea convive como lengua oficial con el criollo (Bickerton, 1975).

Algunos autores, entre ellos Patiño (2002), han acuñado el término de "ciclo de vida" para referirse a la evolución de las lenguas criollas. Dicho término cubre el surgimiento del pidgin por el contacto lingüístico, pasando por la nativización y creación del criollo, hasta llegar a la descriollización o pérdida de los rasgos puramente criollos de estas lenguas al aproximarse a la lengua lexificadora de superestrato. No obstante, esta concepción acerca de la vida de las lenguas criollas ha sido rebatida en los últimos estudios sobre criollos de base léxica inglesa principalmente, incluso llegando a cuestionar la adecuación del continuo. Bickerton (1973) justifica así la existencia del continuo:

After emancipation, the social, political, and economic barriers between whites and non-whites where gradually but progressively weakenedwhile white norms remained, at least until very recently, dominant in the community as a whole. In consequence, a slowly increasing segment of the creole-speaking population was provided with both opportunity and motivation to modify its linguistic behavior in the direction of the approved variety. (p. 644)

Para otros autores como Alleyne (1980) y LaPlage (1977), el escenario descrito por Bickerton no fue el principal creador de la descriollización. Para ambos, las variedades del continuo existieron incluso antes de la emancipación, en periodos primarios de contacto entre los europeos y los africanos en las colonias del nuevo mundo, contrario a lo que pensaba Decamp quien consideraba el continuo como un fenómeno reciente. Esto quiere decir que la variación y los mesolectos existen desde el comienzo, puesto que en las plantaciones existían una variedad de esclavos con un diferente grado de aculturación y uso de la lengua; entre ellos se 
encontraban los esclavos domésticos, los esclavos del campo y un grupo medio de artesanos. Los primeros se aculturaron en mayor grado que los del campo, por su parte el grupo medio estaba en continuo contacto con ambos grupos, haciendo que se creara el mesolecto. De este modo, la criollización no se puede ver como un ciclo de vida pues no necesariamente se pasa de un basilecto a un mesolecto y luego a la descriollización total.

Winford (1997) afirma que el criollo urbano de Guyana no puede ser entendido desde el proceso de descriollización. Este criollo urbano debe ser concebido como un criollo en sí mismo, independiente de la variedad rural, o basilectal. Esta tesis se fundamenta en la forma en que se constituyeron estos dos criollos. Dada la necesidad de importar esclavos para las plantaciones se trajeron habitantes de Antigua, Barbados y San Kits, colonias inglesas, haciendo que entraran en contacto diversas variedades del inglés, unas más criollizadas que otras, con criollos holandeses y franceses de previas colonizaciones, esto originó el criollo rural. Por su parte, el criollo urbano se conformó a partir de los hablantes ingleses, colonizadores, al entrar en contacto con algunos de los hablantes de criollo-inglés de las islas mencionadas, este proceso hizo que en esta zona se formara una variedad reestructurada del inglés. Así, cuando se dio el proceso de posemancipación (1838), los criollos que se trasladaron del campo a la ciudad se vieron en la necesidad de aprender esta última variedad acomodándose a la situación lingüística urbana. Las dos situaciones, la rural y la urbana, presentan diferencias muy marcadas, pues mientras en la primera se presentaba una riqueza lingüística importante, en la segunda el contacto se dio entre hablantes cuya base léxico lingüística fue una sola, el inglés. De este modo, es posible concluir que los criollos hablados en Guyana presentan cada uno una historia particular de surgimiento que los hace independientes y por consiguiente no es posible hablar de un continuo, pues el criollo basilectal, el rural, no dio origen al criollo urbano, anteriormente concebido como mesolectal. Este escenario sugerido para Guyana se ha encontrado, mutatis mutandis, en otras islas del Caribe como Dominica, Grenada, Santa Lucia, San Vicente y Trinidad, haciendo que el concepto de continuo lingüístico poscriollo no pueda generalizarse como lo hizo Bickerton (Winford, 1997).

Por su parte, el criollo trinitario de base léxica inglesa se originó en el siglo xix debido al contacto lingüístico, no solo de lenguas africanas y europeas sino de criollos ya establecidos en el Caribe que llegaron a la isla por asuntos migratorios (James, 2006; Winford, 1997). Luego de la colonización francesa, que dio origen al criollo francés, la isla pasó a ser parte de los británicos, quienes iniciaron la migración de mano de obra barbadense. Estos esclavos poseían un criollo inglés que entro en contacto con el criollo francés; sin embargo, y debido a que los barbadenses empezaron a ocupar los cargos de mayor prestigio, fue este criollo de base léxica inglesa el que se convirtió en el modelo que la población debía alcanzar. De esta manera, muchos de los hablantes del criollo francés tuvieron que reestructurar su lengua y adoptar elementos del inglés criollo, generando una lengua criolla más influida por el criollo barbadense que por el criollo francés trinitario. Así, en Trinidad no se puede hablar de continuo específicamente, pues como sucede en Guyana, las diferentes variedades no se originaron desde un basilecto. Una perspectiva que explica mejor lo que debería ser el continuo en estas situaciones es la creada por Winford donde afirma:

\footnotetext{
Neither one model that posits a line of development from basilec to mesolect to acrolect, nor one that posits the reverse order, seem to me to capture the whole picture of creoles. I view the development of the so-called creole continuum as the result of a multi-faceted array of contact situations producing different outcomes that in true have interacted with each other to produce new varieties or new patterns of variation. (Winford, 1997, p. 254)
}

Desde esta perspectiva, un mejor acercamiento al continuo lingüístico poscriollo es aquel que lo considera como la coexistencia de sistemas que interactúan para producir diferentes variaciones en todos los niveles. Se debe dejar de lado la vieja perspectiva 
Universidad Pedagógica Nacional

Facultad de Humanidades

de que la variación que se da en los países de habla criolla se debe a un proceso natural de descriollización de una lengua vernácula a su respectivo estándar, sería mejor verla como las manifestaciones que surgen debido a un contacto lingüístico que genera dialectos y hablantes bilingües, estos últimos con la capacidad de moverse de una variedad a otra en respuesta a factores como la situación comunicativa, la audiencia, y el contexto discursivo (Winford, 1997).

Una teoría actual que se adecua más a la perspectiva anterior es el denominado varialingüismo de Youssef $(1996,2002,2011)$. Esta teoría se basa en la situación sociolingüística de Trinidad y Tobago y tiene como eje central el individuo y el uso que este hace de los códigos lingüísticos. El varialingüismo es comparable con la competencia que tienen los bilingües y los multilingües de mezclar dos o más códigos en las interacciones orales; sin embargo, se diferencia de esta en la relación que existe entre los códigos en contacto y en el contexto sociolingüístico en que dichos códigos son usados; en el varialingüismo los códigos en contacto comparten una gran parte de su léxico y tienen gramáticas convergentes (Youssef, 1996), debido a esto, los códigos son percibidos como variantes de una misma lengua por los hablantes, haciendo que la interferencia tienda a ocurrir mucho más que en situaciones de bilingüismo normativas. El varialingüismo, entonces, puede ser aplicado a cualquier situación sociolingüística en donde coexisten un criollo y una lengua estándar en una situación de leaking diglosssia (Fasol, 1986), esto es, donde la distribución de funciones se está perdiendo y una variedad empieza a asumir las funciones de la otra. Este es el caso de Trinidad y Tobago, pues el criollo ha estado asumiendo las funciones del estándar y viceversa.

El varialingüismo aparece en Trinidad a partir de la educación y de la lengua que recibe el individuo en el hogar. Un niño que crece en este país, al llegar a la edad escolar, ha estado expuesto a la lengua criolla en el hogar, mesolectal en Trinidad y basilectal en Tobago; luego durante su proceso de alfabetización el inglés estándar es introducido fuertemente como la variedad que se quiere alcanzar, pero sin una demarcación clara con el criollo (Youssef, 2002). El estándar es presentado como una mejor variedad, como la lengua de la educación; no obstante, el criollo es tolerado y debido a que la brecha entre las dos variedades se está angostando, los profesores cada vez tienen menos claro qué pertenece al criollo y qué al estándar. Así, el niño crece aprendiendo a mezclar los códigos en un espacio sociolingüístico multidimensional, aprende a usar las variedades sin diferencia, pero de acuerdo a una plétora de factores situacionales, esto es, el niño es sensible, por ejemplo, al input y responde a él dependiendo del contexto comunicativo (Youssef, 1996, 2001). De este modo, el varialingüismo involucra diferentes cambios de código al igual que diferentes niveles de competencia de los códigos en contacto. Un individuo está difícilmente expuesto al inglés estándar en su totalidad, es decir, no puede adquirir todas sus reglas; esto conlleva a una competencia individual que puede diferir entre individuos debido a su experiencia lingüística y social.

En un estudio denominado The varilingual repertoire of tobagonian speakers, Youssef (2011) analiza el habla de los habitantes de dicha isla y encuentra la denominada competencia varialingual. Dichos hablantes produjeron algo de criollo mesolectal y acrolectal o basilectal de forma controlada; a parte de los hablantes mayores que carecen de educación, todos los participantes de su estudio produjeron basilecto y mesolecto o mesolecto y acrolecto variando intensamente dentro de un solo enunciado. Un ejemplo de uno de los informantes tomado del estudio permite evidenciar dicho fenómeno:

You want a good Bethel twang? Is a mix-up a mix up. If I know you have educated background I my best to speak so up - but when I meet the average people then I does say: "Eh man, wha: go up on a road de:. They does say 'Eh man, eh man, what me a go for? So all these things we ha: to meet up.'

(Youssef, 2011, p. 195)

Para esta autora, esto demuestra que existe una competencia individual dependiente de las situaciones contextuales. Es decir, el varialingüismo es una competencia y no una fase transitoria, como el con- 
tinuo lingüístico poscriollo afirmaba, que le permite al individuo desenvolverse en una sociedad donde coexisten diversos códigos que no necesariamente compiten sino que conviven en los sujetos (Youssef, 2004). De este modo, se debe dejar de percibir el continuo lingüístico poscriollo como el eje central para describir el cambio lingüístico, al menos en el Caribe, pues la escogencia de un código por parte de un individuo, más que deberse a la descriollización, está determinada por parámetros sociales, no solo de la situación comunicativa sino también por aspectos educativos, y por la negociación que se da con el interlocutor.

\section{La criollística como disciplina, orígenes y retos}

En este apartado se hace un breve recuento del origen de la criollística y se presentan los retos que tendrá que afrontar esta disciplina en un futuro próximo. De acuerdo con Baker y Mühlhäusler (2006), la filología no se preocupó por describir ni caracterizar los criollos, pues su objetivo central era la interpretación de documentos literarios e históricos, y dada la ausencia de dichos documentos en lenguas criollas, los filólogos las consideraron como lenguas periféricas. No es sino hasta las primeras misiones que se empiezan a realizar trabajos sobre criollos desde una perspectiva individualista. Los estudios sobre el Sranan (1770) hablado en Surinam y el Negerhollands (1765) hablado en las actuales Islas Vírgenes de los Estados Unidos fueron las primeras aproximaciones serias a una lengua criolla, en este caso de base léxica danesa. En el caso de los criollos franceses fue solo hasta el siguiente siglo que se empezaron a estudiar, las dos investigaciones más elaboradas fueron sobre el criollo francés trinitario (1872) y el criollo de Guyana (1880), en este caso elaborados por dos misioneros: SaintQuentin y Baissac.

Las perspectivas históricocomparativas dominaron el estudio de estas lenguas durante el siglo XIX. Uno de los elementos centrales de los filólogos en ese tiempo era la relación que las lenguas podrían tener entre ellas, representada en un árbol genealógico.
De este modo, las nuevas lenguas eran concebidas como separaciones de las lenguas anteriores, hecho que se denominó como partenogénesis lingüística. Este concepto no permitió concebir la existencia de lenguas mixtas (Baker y Mühlhäusler, 2006). Las lenguas criollas, entonces, fueron vistas más como un elemento irritante y difícil de explicar, enfatizando su "naturaleza aberrante" y negándoseles el estatus de lenguas, esto con el fin de proteger el paradigma partogenético. Por otra parte, el primer estudio comparativo serio de un criollo fue el desarrollado por el especialista bíblico William Greenfield quien se propuso traducir el Nuevo Testamento en Sranan. Esta tarea demostró que las lenguas criollas eran tan complejas y sistemáticas como cualquier otra lengua; sin embargo, su trabajo permaneció olvidado por bastante tiempo (Baker y Mühlhäusler, 2006).

En la segunda mitad del siglo xIx ya existía un número significativo de publicaciones sobre las lenguas criollas principalmente elaboradas a partir de estudios comparativos. Los primeros estudios se enfocaron en caracterizar la gramática de los criollos franceses de Martinica, Trinidad y Santo Tomas. Dichos estudios encontraron elementos paralelos entre las lenguas criollas y sus respectivos lexificadores europeos, hecho que llevo a la generación de la teoría del origen basada en el superestrato (Baker y Mühlhäusler, 2006). A finales del siglo xıx, los estudios se centraron en caracterizar la fonología y la gramática de las lenguas criollas llegando a la conclusión que algunos de los elementos encontrados en estos niveles pertenecían a la lengua de los esclavos, generalmente lenguas africanas, mientras que el léxico a la lengua de los colonizadores. Esto llevó a la futura creación de la teoría sustrastista del origen de las lenguas criollas; algunos estudios trataron de recrear la forma en que los colonizados adquirieron la lengua de sus colonizadores, llevando a la hipótesis, luego desarrollada por Bickerton, de un aprendizaje limitado. Así, al terminar el siglo diecinueve se constituyeron tres ideas centrales sobre las lenguas criollas: (1) las lenguas criollas son formas reducidas de una lengua europea con o sin influencias de esta, perspectiva del superestrato; (2) es posible que las lenguas criollas sean producto 
Universidad Pedagógica Nacional

Facultad de Humanidades

de una adquisición imperfecta de la lengua y (3) las lenguas criollas al no poseer gramáticas europeas deben su origen a las lenguas de sustrato (Baker y Mühlhäusler, 2006).

Durante el siglo xx proliferan los estudios sobre el criollo haitiano y aparecen las teorías de Bloomfield sobre descriollización y nativización anteriormente expuestas. Sin embargo, no es sino hasta la aparición de las memorias del congreso sobre lenguas criollas, llevado a cabo en Jamaica en 1968, que la criollística comienza a verse como una disciplina. A partir de este momento las lenguas criollas se toman como fenómenos de particular interés para la lingüística pues estas pusieron en entre dicho muchos de los postulados hasta ahora establecidos. Tal vez el texto de Hall titulado The Life Cycle of Pidgin Languages (1962) fue el que más debate generó, ya que en él Hall postuló que los pidgins diferían de otras lenguas en su constitución, evolución y desaparición. Después del aporte de Hall surgieron otros estudios, digno de mención es el concepto de bioprograma de Bickerton (1984), que pretendían explicar el origen de las lenguas criollas desde una perspectiva generativa (Baker y Mühlhäusler, 2006). En definitiva, los estudios hasta este momento elaborados permitieron determinar que las lenguas criollas difieren de las lenguas colonizadoras por surgir en circunstancias especiales y por tener características particulares.

Como es evidente, una de las mayores preocupaciones de la criollística ha sido determinar la forma en que los criollos surgieron, se desarrollaron y se convirtieron en lenguas maternas. De acuerdo con Baptista (2005) y Kouwenberg (2011), por más de tres décadas las investigaciones respecto a este tema se dividieron en tres perspectivas antagonistas: la monogenética, la poligenética y la universalista. Sin embargo, estas perspectivas, como se evidenció, solo se centran en uno de los aspectos fundamentales de las lenguas criollas: su no uniformidad, sus orígenes diversos, la poca relación que existe entre algunas de ellas y su historia. Solo hasta la actualidad, los criollistas han dado un giro de la lingüística a la historia con el fin de estudiar la relación entre estos elemen- tos. Para ello han hecho investigaciones diacrónicas y centradas en diferentes países que intentan dilucidar preguntas como ¿qué tipo de población llego a las colonias? ¿En qué época llegaron estas poblaciones? ¿Cuáles fueron las diferentes clases sociales en cada colonia? y ¿Qué tipo de relaciones comerciales existían entre los habitantes y las colonias? Todas ellas, preguntas esenciales para determinar los tipos de comunicación que se llevaron a cabo.

Los procesos cognitivos que se dan en el individuo al momento del surgimiento de un criollo es otra área de bastante importancia que debe ser explorada por los criollistas. Para ello, se deben realizar dos tipos de estudios: (1) investigaciones que comprueben teorías como la relexificación, y la reestructuración gramatical a la luz de los hechos sociales y (2) poner en diálogo los postulados del bilingüismo sobre cambio y mezcla de códigos con teorías como la nativización. Además, es importante que los nuevos estudios realizados sincrónicamente ayuden a determinar las variaciones inter e intralingüísticas que se dan en las lenguas criollas, esto con el fin de determinar los elementos comunes de la criollización, los cambios lingüísticos, los procesos de adquisición y la teoría lingüística (Baptista, 2005).

\section{Conclusiones}

En este texto se hizo evidente que las lenguas criollas son sistemas de comunicación particulares. Además, estas no solo surgieron de manera distinta en comparación con las lenguas europeas, sino que sus estructuras y formas de uso son diferentes. Este hecho, a la par, ha puesto en entre dicho conceptos como la pidginización y la criollización, pues como se evidenció no pueden ser utilizados de forma genérica y deben ser puestos en evidencia a través de investigaciones empíricas. Otro elemento central es que la gran variedad de lenguas criollas en el mundo, especialmente en el Caribe, demuestra que son sistemas de comunicación tan validos como las lenguas dominantes; sin embargo, no se pueden ver desde una sola perspectiva, ni de manera totalizadora, pues cada criollo se debe a situaciones históricas y sociales específicas. Por esto, términos como 
el continuo lingüístico poscriollo y la descriollización deben ser reevaluados por nuevas teorías que se centren en analizar el uso que los hablantes hacen de sus lenguas, como es el caso del varialingüismo. Por otra parte, quedó en evidencia que la criollística debe llevar a cabo investigaciones interdisciplinares para poder determinar cuál fue el origen de estas lenguas, pues posturas centradas en un solo elemento limitan el entendimiento de este fenómeno.

\section{Referencias}

Aceto, M. (1999). looking beyond decreolization as an explanatory model of language change in creolespeaking communities. Journal of Pidgin and Creole Languages, 14(1), 93-119.

Aceto, M. (2006). Statian Creole English: an English-derived language emerges in the Dutch Antilles. World Englishes, 25(3/4), 411-435. doi: 10.1111/j.1467-971X.2006.00480.x

Alleyne, M. (1980). Comparative afro-american: A historical-comparative study of english-based afro-american dialects of the new world. An Arbor: Karoma.

Alleyne, M. (1994). Problems of Standarization of Creole languages. In M. Morgan (Ed.), Language and the social construction of identity in Creole situations (pp. 7-18). USA: Center for Afro-American Studies. UCLA.

Arends, J. (1995). The socio-historical background of creoles. In J. Arends (Ed.), Pidgins and Creoles (pp. 15-24). USA: John Benjamins.

Bailey, B. (1966). Jamaican Creole Syntax. Cambridge: Cambridge University Press.

Baker, D., \& Mühlhäusler, P. (2006). Creole Linguistics from its beginnings, through Schuchardt to the present In C. Stewart (Ed.), Creolization : History, Ethnography, Theory. USA: Left Coast Press.

Bakker, P. (2008). Pidgins versus Creoles and Pidgincreoles. In S. Kouwenber \& J. Singler (Eds.), The Handbook of pidgin and creoles studies (pp. 132-157). Singapore: Wiley-Blackwell.

Baptista, M. (2005). New Directions in Pidgin and Creole Studies. Annual Review of Anthropology, 34(1), 33-42. doi: 10.1146/annurev.anthro.34.081804.120638

Bickerton, D. (1973). The Nature of a Creole Continuum. Language, 49, 640-669.
Bickerton, D. (1975). Dynamics of a creole system. Cambridge: Cambridge University Press.

Bickerton, D. (1976). Pidgin and Creole Studies. Annual Review of Anthropology, 5, 169-193.

Bickerton, D. (1981). Roots of languages. . London: Ann Arbor Karoma Publishers.

Bickerton, D. (1984). The language bioprogram hypotesis. Brain Behav. Sci, 7, 173-221.

Chaudenson, R. ( 1977). Toward the reconstruction of social matrix of Creole languages. In A. Valdam (Ed.), Pidgin and Creole Linguistics. USA: Indiana University Press.

Decamp, D. (1971). Introduction: The study of pidgin and creole languages. In D. Hymes (Ed.), Pidginization and creolization of languages. Proceedings of a conference held at The University of the West Inides Mona, Jamiaca, April 1968 (pp. 13-39). London: Cambridge University Press.

Doucet, R. (2000). Creole language instruction in Haiti. Paper presented at the Fifth International Creole Language Workshop, Florida International University.

Fasol, R. (1986). The sociolinguistics of language. Oxford: Oxford University Press.

Hancock, I. (1971). A provisional comparison of the English-derived Atlantic creoles. In D. Hymes (Ed.), Pidginization and creolization of languages. Proceedings of a conference held at The University of the West Inides Mona, Jamiaca, April 1968 (pp. 287291). London: Cambridge University Press.

Hall, R. (1962). The life cycle of pidgin languages. Lingua, 11, 151-156.

Herzfeld, A. (2002). Mekaytelyuw. Costa Rica: Editorial de la Universidad de Costa Rica.

Herzfeld, A. (2008). Los criollos en Centroamérica. In A. Palacios (Ed.), El español en América Contactos lingüísticos en Hispanoamérica (pp. 77-94). Barcelona: Ariel.

Holm, J. (2000). Introduction to Pidgins and Creoles. USA: Cambridge University Press.

Holm, J. (1989). Pidgins and Creoles (Vol. II). Cambridge: Cambridge University Press.

Hymes, D. (1971). Preface. In D. Hymes (Ed.), Pidginization and creolization of languages. Proceedings of a conference held at The University of the West Inides Mona, Jamiaca, April 1968 (pp. 3-11). London: Cambridge University Press. 
Universidad Pedagógica Nacional

Facultad de Humanidades

James, W. (2006). Phonological signatures of tobagonian speech in Trinidad and Tobago. In H. SimmonsMcDonald \& I. Robertson (Eds.), Exploring the boundaries of Caribbean Creole languages (pp. 50-71). USA: University of the West Indies Press.

Jourdan, C. (1991). Pidgins and creoles: the blurring of categories. Annual Review of Anthropology, 20, 187-209.

Kouwenberg, S. (2011). Linguistics in the Caribbean: Between theory and practice. Journal of Pidgin \& Creole Languages, 26(1), 219-233. doi: 10.1075/ jpcl.26.1.08kou

LaPlage, R. (1977). Jamaican creole. USA: The University of Alabama Press.

McWhorter, J. (1998). Identifying the Creole prototype: vindicating a typological class. Language, 74(4), 788-818.

Migge, B. (2003). Creole Formation as Language Contact. The case of the Suriname Creoles. London Benjamins Publishing Company.

Migge, Bettina, Léglise, Isabelle, \& Bartens, Anglea. (2010). Creoles in Education. A discussion of pertinent issues. In B. Migge, I. Léglise \& A. Bartens (Eds.), Creoles in Education. An appraisal of current programs and projects (pp. 1-30). USA: John Benjamins.

Mintz, S. (1971). The socio-historical background to pidginization and creolization. In D. Hymes (Ed.), Pidginization and creolization of languages. Proceedings of a conference held at The University of the West Inides Mona, Jamiaca, April 1968 (pp. 481496). London: Cambridge University Press.

Muysken, P. (1981). Half-way between Quechua and Spanish: the case for relexification. In R. Highfield \& A. Valdman (Eds.), Historicity and Variation in Creole Studies. MI: Karoma.

Muysken, P., \& Veenstra, T. (1995). Haiti. In J. Arends (Ed.), Pidgins and Creoles (pp. 153-164). USA: John Benjamins.

Patiño, C. (2000). La criollística y las lenguas criollas de Colombia. Sobre etnolingüística y otros temas. Bogotá: Instituto Caro y Cuervo.

Patiño, C. (2000a). Las lenguas criollas y la herencia lingüística africana. Sobre etnolingüística y otros temas Bogotá: Instituto Caro y Cuervo.

Patiño, C. (2000b). Sobre etnolingüística y otro temas. Bogotá: Instituto Caro y Cuervo.
Patiño, C. (2002). Historia y sociedad en la genesis de las lenguas criollas. Revista de Estudios Sociales (13), 109-115.

Romaine, S. (1988). Pidgins and Creoles. New York: Longman.

Romaine, S. (1996). El lenguaje en la sociedad. Una introducción a la sociolingüística. Barcelona: Ariel.

Salikoko, S. (2006). Pidgins and creoles. In B. Kachru, Y. Kachru \& C. Nelson (Eds.), The handbook of world Englishes. UK: Blackwell Publishing Ltd.

Siegel, Jeff. (2006). Keeping Creoles and Dialects out of the Classroom: is it justified? In S. Nero (Ed.), Dialects, Englishes, Creoles, and Education (pp. 39-67). London: Lawrence Erlbaum Associates Publishers.

Siguan, M. (2001). Bilinguismo y lenguas en contacto. Madrid: Alianza Editorial.

Veenstra, T. (2008). Creole Genesis: The Impact of the Language Bioprogram Hypothesis. In S. Kouwenber \& J. Singler (Eds.), The Handbook of pidgin and creoles studies (pp. 219-241). Singapore: Wiley-Blackwell.

Weinreich, Uriel, \& Martinet, André. (1963). Languages in contact findings and problems. The Hague: Mouton.

Winford, D. (1997). Re-examining Caribbean Englishes Creole Continua. World Englishes, 16(2), 233-279.

Winford, D. (1994). Sociolinguistic Approaches to Language use in the Caribben. In M. Morgan (Ed.), Language and the social construction of identity in Creole situations (pp. 43-62). USA: Center for AfroAmerican Studies. UCLA.

Youssef, V. (1996). Varilingualism: The competence underlying code - mixing in Trinidad and Tobago. Journal of Pidgin and Creole Languages, 11(1), 1-22.

Youssef, V. (2002). Case Study Issues of Bilingual Education in the Caribbean: The Cases of Haiti, and Trinidad and Tobago. International Journal of Bilingual Education and Bilingualism., 5(3), 82-193.

Youssef, V. (2004). "Is english we speaking”: Trinbagonian in the twenty-first century. English Today, 20(4), 42-49.

Youssef, V. (2011). The Varilingual repertoire of Tobagonian speakers. In L. Hinrichs \& J. Farquharson (Eds.), Variation in the Caribbean: From Creole continua to individual agency (pp. 191-206). USA: John Benjamins.

Youssef, V. (2001). Age-grading in the Anglophone Creole of Tobago. World Englishes, 20(1), 29. 